\title{
A Finite-dimensional Canonical Formalism in the Classical Field Theory
}

\author{
Jerzy Kijowski \\ Institute of Mathematical Methods of Physics, Warsaw University, Warsaw, Poland
}

Received March 8; in revised form November 1, 1972

\begin{abstract}
A canonical formalism based on the geometrical approach to the calculus of variations is given. The notion of multi-phase space is introduced which enables to define whole the canonical structure (physical quantities, Poisson bracket, canonical fields) without use of functional derivatives. All definitions are of pure geometrical (finite dimensional) character.

The observable algebra $\mathcal{O}$ (physical quantities algebra) obtained here is much smaller then the algebra of all (sufficiently smooth) functionals on the space of states, derived from the standard infinite-dimensional formulation. As it is known, the latter is much too large for purposes of quantization. As the examples prove, our algebra $\mathcal{O}$ could be an adequate start-point for quantization.

For simplifying the language the notion of observable-valued distribution is introduced. Many concrete physical examples are given. E.g. it is shown that some problems connected with gauge in electrodynamics are automatically solved in this approach. The introduced language allows to obtain the Noether theorem in a most natural way.
\end{abstract}

\section{Introduction}

The present state of quantization of non-linear fields theories (cf. [1]) may lead to the conclusion that there may be more deep differences between linear and non-linear theories that one may infer from the usual canonical formalism, based on the following analogy with classical mechanics:

\begin{tabular}{ll}
\hline Mechanics & Field theory \\
\hline Time & Time \\
$\begin{array}{l}\text { Finite dimensional space } \\
\text { of all possible positions at given time }\end{array}$ & $\begin{array}{l}\text { Infinite dimensional space } \\
\text { of states of a field at given time }\end{array}$ \\
\hline
\end{tabular}

This formalism, initially using notions not too precise from the mathematical viewpoint (e.g. multiplication of functional derivatives) has now acquired a fine mathematical formulation (theory of infinite dimensional symplectic spaces - cf. [11] and [12]). It leads to very rich observable algebra (algebra of physical quantities), Poisson bracket, canonical fields etc. Linear theories are not distinguished in this formalism. 
Yet another formulation of canonical formalism is possible. It arises from the geometrical approach to the calculus of variations (as presented in the excellent paper by Dedecker [6]). Here another analogy with mechanics is used:

\begin{tabular}{ll}
\hline Mechanics & Field theory \\
\hline Time as 1-dimensional parameter & $\begin{array}{l}\text { 4-dimensional space-time as the space of } \\
\text { parameters }\end{array}$ \\
$\begin{array}{l}\text { Finite dimensional space of positions } \\
\text { and momenta at given time }\end{array}$ & $\begin{array}{l}\text { Finite dimensional space of field strengths and } \\
\text { its derivatives in given point of space-time }\end{array}$ \\
\hline
\end{tabular}

That this approach could be physically relevant it was already noticed several years ago ([15]) but there were serious difficulties in further development of the theory which leads to highly-dimensional spaces. It seems more difficult to work in 77 dimensions (as Dedecker's theory for vector-field theory requires) than in infinite-dimensional space.

Recently very adequate for field theories simplifications of the Dedecker's formulation have been done (these results will be published elsewhere). Using them a beautiful geometrical structure of field theories has been discovered.

In the present paper we leave aside the connection between Lagrangean and Hamiltonian formalism (i.e. the Dedecker's theory) and we present the theory in its "canonical" form.

Our construction leads at last to a triplet $(\mathscr{H}, \mathcal{O}, \mathscr{Z})$ where:

$1^{\circ} \mathscr{H}$ is (already infinite dimensional) space of states (whose elements are global solutions of field equations).

$2^{\circ}$ ( ) is a Lie algebra of observables (physical quantities), i.e. an algebra of very special functionals on $\mathscr{H}$. It is composed of such functionals which are integrals of some differential forms. This restriction of "admissible functionals" is not arbitrary - it results from the language we use (the accepted here definition of observable is very natural one). Curious as might seem a very natural functional characteristic of our observable algebra has been found ([10]): it is the algebra of local functionals on $\mathscr{H}$ (of some degree of smoothness).

$3^{\circ} \mathscr{Z}$ is a special vector field subalgebra on $\mathscr{H}$. Its elements are, in some way, generated by observables.

The advantages of such approach seem to be as follows:

1. Our observable algebra is very small in comparison with the usual algebra of all smooth functionals on $\mathscr{H}$. In quantization, as it is known, only few observables can be quantized directly. So the question arises: "which Lie subalgebra of observables can be directly represented in Hilbert space?" (cf. [4]). Our formulation answers this question in a 
surprisingly simple way. E.g. for the Klein-Gordon theory the only observables are: generators of Poincaré group, field-strength and its time-derivative (smeared with an arbitrary test-function). There are no other observables.

2. The only algebraic structure of our observable algebra is a Lie structure given by Poisson bracket. There is no commutative algebra structure (there is no sense multiplying observables). So there is no question of "good order of operators" during quantization.

3. There are no difficulties with the gauge in electrodynamics. Only electric and magnetic fields (smeared with test functions) are observables (cf. [3]). Potentials are not observables (see Chapter 8).

4. The most interesting result of this theory is at the same time its greatest drawback now: in non-linear theories there are no local observables. The only observables are the global ones (i.e. energy, momentum, electric charge etc. - see Appendix).

Thus there seem to be two ways out:

1. Either the solution suggested by [10] (introduction of higherorder currents) will enable construction of satisfactory algebra of local observables for non-linear theories (which may prove to be of considerable importance for comprehension of non-linear quantization)

2. or such satisfactory construction is not to be obtained at all. This may be connected with impossibility of quantization in non-linear cases.

As yet the solution of this problem has not been known to the author.

The question of equal-time Poisson bracket has already been satisfactorily solved by Gawędzki in an ingenious paper [8].

The author is much indebted to Dr. W. Tulczyjew (who first used the notion "multiphase-space") and Prof. Dr. I. Birula-Białynicki for very fruitful discussions.

Many problems touched here were studied in the collaboration with Dr. K. Gawędzki, for which I thank him very much.

Special thanks are due to Prof. Dr. K. Maurin for his encouragements and active interest in this work.

\section{Homogeneous Formalism in the Classical Mechanics}

Homogeneous formalism, based on geometrical concepts of Cartan, was used already in twenties. We shall present it in a most useful version, showing these elements which look similar in the field theory (cf. $[15,16]$ ).

Let $W$ be a full (i.e. containing time-coordinate) configuration space of mechanical system. Take any coordinate chart $\left(t, x^{i}\right), i=1, \ldots, k$, where $t$ denotes time and $x^{i}$ are any coordinates numbering all possible positions of our system (e.g. for the theory of single point-like particle 
$k=3$ and $W$ is simply space-time). Denote by

$$
T^{*}(W)=\bigcup_{w \in W} T_{w}^{*}(W)
$$

the co-tangent bundle over $W$ (composed of all co-tangent spaces $T_{w}^{*}(W)$, at all points $\left.w \in W\right)$. Every element of $T^{*}(W)$ can be written in the form

$$
E d t-\Sigma p_{i} d x^{i}
$$

thus we can take in $T^{*}(W)$ the coordinate chart $\left(t, x^{i}, E, p_{i}\right)$. There are canonical differential forms in the space $T^{*}(W)$ :

$$
\begin{aligned}
\omega\left(t, x^{i}, E, p_{i}\right) & =E d t-\Sigma p_{i} d x^{i} \\
d \omega\left(t, x^{i}, E, p_{i}\right) & =d E \wedge d t-\Sigma d p_{i} \wedge d x^{i}
\end{aligned}
$$

Take in $T^{*}(W)$ the submanifold $\mathscr{P}$ given by the equality $E=H\left(t, x^{i}, p_{i}\right)$, where $H$ is a smooth function of its arguments. If $H$ is Hamiltonian of our system then $\mathscr{P}$ will be called the full phase space of it. $\mathscr{P}$ is $(2 k+1)$ dimensional and can be parametrized by $\left(t, x^{l}, p_{i}\right)$.

Take the cut-off of the 2-form $d \omega$ to the space $\mathscr{P}$ :

$$
\begin{aligned}
\gamma:=d \omega \mid \mathscr{P} & =d H\left(t, x^{i}, p_{i}\right) \wedge d t-\Sigma d p_{i} \wedge d x^{i} \\
& =\Sigma\left(\frac{\partial H}{\partial x^{i}} d x^{i} \wedge d t+\frac{\partial H}{\partial p_{i}} d p_{i} \wedge d t-d p_{i} \wedge d x^{i}\right) .
\end{aligned}
$$

Of course $d \gamma=d^{2} \omega \mid \mathscr{P}=0$. The form $\gamma$ is degenerated. It can be shown that singular curves of $\gamma$ (i.e. such curves whose tangent vector is everywhere singular vector for $\gamma$ ) are exactly solutions of the dynamical problem with the Hamiltonian $H$ :

Let $\Omega$ be such a curve (for shortening the language such curves will be called states). Take any parametrization of $\Omega$ :

$$
\tau \rightarrow\left(t(\tau), X^{i}(\tau), p_{i}(\tau)\right) .
$$

Let the parametrization be non-singular, i.e.

$$
X=\frac{d t}{d \tau} \frac{\partial}{\partial t}+\Sigma\left\{\frac{d x i}{d \tau} \frac{\partial}{\partial x^{i}}+\frac{d p_{i}}{d \tau} \frac{\partial}{\partial p_{i}}\right\} \neq 0 .
$$

Then one can easily compute:

$$
\begin{aligned}
X\lrcorner \gamma= & \frac{d t}{d \tau}\left(\Sigma \frac{\partial H}{\partial x^{i}} d x^{i}+\frac{\partial H}{\partial p_{i}} d p_{i}\right)+\Sigma \frac{d x^{i}}{d \tau}\left(-\frac{\partial H}{\partial x^{i}} d t-d p_{i}\right) \\
& +\Sigma \frac{d p_{i}}{d \tau}\left(-\frac{\partial H}{\partial p_{i}} d t+d x^{i}\right)=-\left(\Sigma \frac{\partial H}{\partial x^{i}} \frac{d x^{i}}{d \tau}+\frac{\partial H}{\partial p_{i}} \frac{d p_{i}}{d \tau}\right) d t \\
& +\Sigma\left(\frac{\partial H}{\partial x^{i}} \frac{d t}{d \tau}+\frac{d p_{l}}{d \tau}\right) d x^{i}+\Sigma\left(\frac{\partial H}{\partial p_{i}} \frac{d t}{d \tau}-\frac{d x^{i}}{d \tau}\right) d p_{i}=0 .
\end{aligned}
$$


In order to satisfy this equality all coefficients in brackets must vanish. But in this case the vanishing of the derivative $\frac{d t}{d \tau}$ implies the vanishing of $X$. So $\frac{d t}{d \tau} \neq 0$. Thus in order to simplify the equation of motion we can take $\tau=t$. Now $\frac{d t}{d \tau}=1$ and the Eq. (1) reads:

$$
\begin{gathered}
\frac{\partial H}{\partial x^{l}}+\frac{d p_{i}}{d t}=0, \quad \frac{\partial H}{\partial p_{\imath}}-\frac{d x^{i}}{d t}=0, \\
\frac{\partial H}{\partial x^{i}} \frac{d x^{l}}{d t}+\frac{\partial H}{\partial p_{i}} \frac{d p_{i}}{d t}=0 .
\end{gathered}
$$

If e.g. $H=\frac{1}{2 m}\left(\Sigma p_{\imath} p_{i}\right)+V(x)$ then

$$
\frac{d x^{i}}{d t}=\frac{1}{m} p_{i} \quad\left(\text { it means that velocity is equal } \frac{1}{m} p_{i}\right) .
$$

The Eqs. (2) are the canonical equations of Hamilton. The third one is the consequence of them. It is the energy-equation: adding to both sides of (3) the term $\frac{\partial H}{\partial t}$ we obtain

$$
\frac{d E}{d t}:=\frac{d H}{d t}=\frac{\partial H}{\partial t} .
$$

In relativistic mechanics, when we use the Minkowski's metric tensor with the sygnature $(+,-,-,-)$ we want to have $v^{i}=\frac{1}{m} p^{i}$ and $p^{i}=-p_{i}$. It is therefore much better to use the following notation:

$$
\omega=\sum_{\mu=0}^{3} p_{\mu} d x^{\mu}=E d t+\sum_{i=1}^{3} p_{i} d x^{l}=E d t-\sum_{i=1}^{3} p^{i} d x^{i}
$$

where $p_{0}=p^{0}=E, x^{0}=x_{0}=t$. In this notation canonical equations of motion are

$$
\frac{d x^{i}}{d t}=-\frac{\partial H}{\partial p_{i}}, \quad \frac{d p_{1}}{d t}=\frac{\partial H}{\partial x^{i}} .
$$

Taking e.g. as $\mathscr{P}$ the submanifold satisfying equation $p^{\mu} p_{\mu}=m^{2}, p_{0}>0$ (i.e. $p_{0}=H=\sqrt{m^{2}+\Sigma p_{i} p_{i}}$ ) we obtain

$$
\frac{d x^{l}}{d t}=-\frac{\partial H}{\partial p_{\imath}}=-\frac{p_{i}}{E}=\frac{p^{i}}{E} \quad \frac{d p_{i}}{d t}=0 .
$$


Such an approach is called "homogeneous" because there is no difference between time and another coordinates. There is no "motion" in this picture, but only singular curves. The whole "dynamics" is given by the phase space $\mathscr{P}$ and 2 -form $\gamma$.

Now we pass to physical quantities (observables). They are such functions (i.e. differential 0 -forms) on the space $\mathscr{P}$, which are constant on states (singular curves of $\gamma$ ). In this approach "position of a particle" is not an observable, but "position at time $t_{0}$ " is. Take e.g. the theory of single relativistic particle, as presented above. States are here straight lines:

$$
t \rightarrow\left(t, x_{0}^{i}+\frac{p^{i}}{E}\left(t-t_{0}\right), p_{j}\right) .
$$

The observable "position at $t_{0}$ " now reads:

$$
x_{t_{0}}^{i}\left(t, x^{j}, p_{j}\right)=x^{i}-\frac{p^{i}}{E}\left(t-t_{0}\right) .
$$

The function $p_{j}$ is also an observable because in this case the momentum is a constant of motion.

It can be shown that the function $f$ on $\mathscr{P}$ is an observable if and only if

$$
d f=X\lrcorner \gamma
$$

where $X$ is a vector field. But we have immediately:

$$
\left.\left.\left.0=d^{2} f=d(X\lrcorner \gamma\right)=-\{X\lrcorner d \gamma-d(X\lrcorner \gamma\right)\right\}=-\mathscr{L}_{X} \gamma,
$$

i.e. the field $X$ preserves $\gamma$ (the one-parameter group of diffeomorphisms generated by $X$ preserves $\gamma$ ). Such fields are called canonical ones.

If we have two observables $f$ and $g$ :

$$
d f=X\lrcorner \gamma, \quad d g=Y\lrcorner \gamma
$$

then the Poisson bracket

$$
\{f, g\}=\mathscr{L}_{X} g
$$

is also an observable. This definition is completely equivalent to the usual one. E.g. in the last example

$$
\left.\left.d p_{j}=\frac{\partial}{\partial x^{j}}\right\lrcorner \gamma, \quad d p_{0}=\frac{\partial}{\partial t}\right\lrcorner \gamma .
$$

Thus

$$
\begin{aligned}
& \left\{p_{j}, x_{t_{0}}^{i}\right\}=\frac{\partial}{\partial x^{j}}\left(x_{t_{0}}^{i}\right)=\frac{\partial}{\partial x^{j}}\left(x^{i}-\frac{p^{i}}{E}\left(t-t_{0}\right)\right)=\delta_{j}^{i} \\
& \left\{p_{0}, x_{t_{0}}^{i}\right\}=\frac{\partial}{\partial t}\left(x_{t_{0}}^{i}\right)=-\frac{p^{i}}{E} .
\end{aligned}
$$


Let us do at last the following construction.

Denote by $D^{0}$ the distribution (in the geometrical meaning of this word) of all singular for $\gamma$ subspaces tangent to $\mathscr{P}$. As we have seen $D^{0}$ is here 1-dimensional and solutions of our mechanical problem are also integral curves for $D^{0}$. Thus we can pass to the quotient $\mathscr{P}^{\prime}=\mathscr{P} / D^{0}$. Elements of $\mathscr{P}^{\prime}$ are curves in $\mathscr{P}$. Because every such a curve pierce the subspace $t=$ const at precise one point, there is one-to-one correspondence between points of $\mathscr{P}^{\prime}$ and points of the subspace $t=$ const. It means, that if we fix the time the system $\left(x^{i}, p_{i}\right)$ is a good coordinate chart in $\mathscr{P}^{\prime}$. Because $D^{0}$ is singular for $\gamma$, we can project $\gamma$ to the form $\gamma^{\prime}$ on $\mathscr{P}^{\prime}$. This last form is already non-degenerated (whole degeneration lies in $D^{0}$ ). In coordinate chart $\left(x^{i}, p_{i}\right)$, connected with the fixed time $t$, the form $\gamma^{\prime}$ can be written as

$$
\gamma^{\prime}=-\Sigma d p_{i} \wedge d x^{l}
$$

\section{General Scheme of Multi-phase Formalism. Gauge}

Leaving aside the problem of concrete method of constructing the phase space for concrete field equation (cf. examples in Chapter 4 and 8 ) we shall formulate the "axiomatic" canonical theory, analogous to the homogeneous formulation of mechanics. We take the following

Definition. By the $n$-phase space we mean the couple $(\mathscr{P}, \gamma)$ where $\mathscr{P}$ is $m$-dimensional $(m>n)$ countable at infinity, smooth manifold (by smoothness we shall always mean that of $C^{\infty}$-class); $\gamma$ is an $(n+1)$ differential closed form (i.e. $d \gamma=0$ ) in $\mathscr{P}$.

The form $\gamma$ defines the mapping

$$
\left.T_{p}(\mathscr{P}) \ni X_{p} \rightarrow X_{p}^{*}:=X_{p}\right\lrcorner \gamma_{p} \in \bigwedge^{n} T_{p}^{*}(\mathscr{P})
$$

(by $\bigwedge^{n} T_{p}^{*}(\mathscr{P})$ we mean the $n$-th exterior power of the cotangent space $T_{p}^{*}(\mathscr{P})$, i.e. the space of $n$-covectors at $\left.p\right)$.

Every vector field $X$ in $\mathscr{P}$ defines therefore the $n$-form $X^{*}$. For every $p \in \mathscr{P}$ we distinguish the subspace of $n$-covectors obtained in this way:

$$
\mathscr{A}_{p}:=T_{p}(\mathscr{P})-\gamma_{p} \bigwedge^{n} T_{p}^{*}(\mathscr{P})
$$

Now we seek the maximal tangent subspaces anihilating $\mathscr{A}_{p}$. The family of all such subspaces at $p \in \mathscr{P}$ will be denoted $\mathscr{A}_{p}^{\prime}$. So

$$
\left(E \in \mathscr{A}_{p}^{\prime}\right) \Rightarrow\left(\alpha \mid E=0 \quad \text { for every } \quad \alpha \in \mathscr{A}_{p}\right) .
$$


Now submanifolds whose tangent spaces belong to $\mathscr{A}_{p}^{\prime}$ are of interest. For the field theory or mechanics such submanifolds will be actually solutions of the motion equations. So they are states of the field or mechanical system. The notion "state" is used here in the sense Einstein applied it - state means the whole history of the system. In quantum physics such a point of view corresponds to the Heisenberg picture.

The following definition seems to be natural:

Definition. By the state in the $n$-phase space $(\mathscr{P}, \gamma)$ we mean any maximal submanifold having property that every its tangent space belongs to $\mathscr{A}^{\prime}$. The set of all states of a given theory will be denoted $\mathscr{H}(\mathscr{P}, \gamma)$ or simply $\mathscr{H}$.

One can easily check that states are maximal submanifolds in $\mathscr{P}$ having the following property:

$$
v\lrcorner \gamma=0
$$

for every $n$-vector field $v$ tangent to this submanifold.

In order to omit pathological cases we take the

Definition. The $n$-phase space $(\mathscr{P}, \gamma)$ is called regular one if all dimensions of all spaces belonging to $\mathscr{A}_{p}^{\prime}$ are equal for all $p \in \mathscr{P}$. This common dimension will be denoted $\operatorname{dim} \mathscr{A}$.

From now on we shall always assume the regularity of $(\mathscr{P}, \gamma)$. Thus $\operatorname{dim} \Omega=\operatorname{dim} \mathscr{A}$ for every state $\Omega \in \mathscr{H}$. tion:

The canonical structure of $(\mathscr{P}, \gamma)$ distinguishes the following distribu-

$$
\left.D^{0}:=\left\{X_{p} \in T_{p}(\mathscr{P}): X_{p}\right\lrcorner \gamma_{p}=0\right\} .
$$

It will be called the primitive gauge of the theory. The primitive gauge fields (i.e. fields belonging to this distribution) are precisely such fields for which $X^{*}=0$.

Observe that $D_{p}^{0} \subset E$ for any $E \in \mathscr{A}_{p}^{\prime}$. Thus $D_{p}^{0}$ is tangent to every state passing by $p$.

Lemma 1. The primitive gauge distribution is involutive, i.e. if $X, Y \in D^{0}$ then $[X, Y] \in D^{0}$ (by $[X, Y]$ we denote the Lie bracket of fields $X$ and $Y$ ).

$$
\text { Proof. } \begin{aligned}
{[X, Y]\lrcorner \gamma } & \left.\left.=\left(\mathscr{L}_{X} Y\right)\right\lrcorner \gamma=\mathscr{L}_{X}(Y\lrcorner \gamma\right)-Y\left(\mathscr{L}_{X} \gamma\right) \\
& =0-Y\lrcorner(X\lrcorner d \gamma-d(X\lrcorner \gamma)) \cdot(-1)^{n+1}=0{ }^{1} .
\end{aligned}
$$

Thus (locally) there exists an integral congruence of $D^{0}$. If it exists globally then we can pass to the quotient space $\mathscr{P}^{\prime}=\mathscr{P} / D^{0}$.

1 We use the following interior product: $\langle u, X\lrcorner \alpha\rangle=\langle u \wedge X, \alpha\rangle$. In this convention the Lie derivative is: $\left.\left.\mathscr{L}_{X} \alpha=(-1)^{m}\{X\lrcorner d \alpha-d(X\lrcorner \alpha\right)\right\}$ if $\alpha$ is an $m$-form (cf. definition of Lie derivative in [5] and [13]). 
In virtue of $\gamma \mid D^{0}=0$ the form $\gamma$ can be projected to the $n$-form $\gamma^{\prime}$ on $\mathscr{P}^{\prime}$. We can thus construct a new $n$-phase space $\left(\mathscr{P}^{\prime}, \gamma^{\prime}\right)$ without any primitive gauge (cf. [15] and [16]).

By full gauge of $(\mathscr{P}, \gamma)$ we mean the distribution $D$ given by the formula

Of course $D^{0} C D$.

$$
D_{p}=\bigcap_{\substack{p \in \Omega \\ \Omega \in \mathscr{H}}} T_{p}(\Omega) .
$$

From the definition one can easily conclude that $D$ is also involutive. But we cannot pass to the quotient because $\gamma$ cannot, in general, be uniquely projected on the quotient space. In this case we can rather take any concrete gauge, i.e. any submanifold transversal to the gauge, covering the whole quotient space (in the calculus of variations e.g. classical theories of Caratheodory or Lepage can be obtained by taking the concrete gauge in the full Dedecker's space. This result will be published elsewhere).

\section{Examples of Multi-Phase Spaces}

1. Phase Space (1-phase Space) in Mechanics - as in Chapter 2.

2. Mechanics with Additional Gauge. Take $W_{g}=W \times R$, where $W$ is taken as in Chapter 2.

Using the coordinates $\left(t, x^{l}, \varphi\right)$ we take

$$
\gamma=d E \wedge d t-\Sigma d p_{i} \wedge d x^{i}+d q \wedge d \varphi
$$

in the submanifold $\mathscr{P} \subset T^{*}\left(W_{g}\right)$ given by the conditions:

$$
\left\{\begin{array}{c}
E=H\left(t, x^{i}, p_{i}\right) \\
q=Q=\text { const }
\end{array}\right.
$$

In local coordinates $\left(t, x^{i}, \varphi, p_{i}\right)$ in $\mathscr{P}$ the space $D_{p}^{0}$ is now spanned by vectors $\frac{\partial}{\partial \varphi}$ and $\frac{\partial}{\partial t}+\frac{\partial H}{\partial p_{i}} \frac{\partial}{\partial x^{i}}-\frac{\partial H}{\partial x^{i}} \frac{\partial}{\partial p_{i}}$. States are now 2-dimensional. The reduced phase space $\mathscr{P}^{\prime}$ is equal to that of Example 1.

3. Scalar Field Theory. $W=M \times R$ with coordinates $\left(x^{0}, x^{1}, x^{2}, x^{3}, \varphi\right)$, $n=4$ (the coordinate $\varphi$ will describe the strength of the field).

From now on we shall often use the Einstein's summation-convention.

In the space $\bigwedge^{4} T^{*}(W)$ there are following canonical forms:

$$
\begin{gathered}
\omega=\eta d x^{0} \wedge \cdots \wedge d x^{3}+\eta^{\mu} d x^{0} \wedge \cdots \wedge d \hat{\wedge}_{\mu} \wedge \wedge \wedge d x^{3} \\
d \omega=d \eta \wedge d x^{0} \wedge \cdots \wedge d x^{3}+d \eta^{\mu} \wedge d x^{0} \wedge \cdots \wedge d \varphi \wedge{ }_{\mu} \wedge \wedge \wedge d x^{3} \\
\text { (by } \wedge \text { we denote the } \mu^{\prime} \text { th place). }
\end{gathered}
$$


As $\mathscr{P} \subset \bigwedge^{4} T^{*}(W)$ take the submanifold given by the formula

$$
\eta=H\left(x^{\mu}, \varphi, \eta^{\mu}\right)
$$

and

$\gamma=d \omega \mid \mathscr{P}=d H \wedge d x^{0} \wedge \cdots \wedge d x^{3}+d \eta^{\mu} \wedge d x^{0} \wedge \cdots \wedge \underset{\wedge}{d} \varphi \wedge \cdots \wedge d x^{3}$

$=\left(\frac{\partial H}{\partial \varphi} d \varphi+\frac{\partial H}{\partial \eta^{\mu}} d \eta^{\mu}\right) \wedge d x^{0} \wedge \cdots \wedge d x^{3}+d \eta^{\mu} \wedge d x^{0} \wedge \cdots \wedge \underset{\mu}{d} \varphi \wedge \cdots \wedge d x^{3}$.

In similar way as in mechanics one can show that every state $\Omega$ can be parametrized by space-time coordinates $x^{\mu}$ :

$$
\left(x^{\mu}\right) \rightarrow\left(x^{\mu}, \varphi\left(x^{\mu}\right), \eta^{\nu}\left(x^{\mu}\right)\right) .
$$

The 4-vector tangent to $\Omega$ is equal $v=X_{0} \wedge X_{1} \wedge X_{2} \wedge X_{3}$, where

$$
X_{\mu}=\frac{\partial}{\partial x^{\mu}}+\frac{\partial \varphi}{\partial x^{\mu}} \frac{\partial}{\partial \varphi}+\frac{\partial \eta^{\nu}}{\partial x^{\mu}} \frac{\partial}{\partial \eta^{v}} .
$$

The equation of motion can be easily computed:

$$
\begin{aligned}
v\lrcorner \gamma= & \frac{\partial H}{\partial \varphi} d \varphi+\frac{\partial H}{\partial \eta^{\mu}} d \eta^{\mu}-\frac{\partial H}{\partial \varphi} \frac{\partial \varphi}{\partial x^{\mu}} d x^{\mu}+\frac{\partial \varphi}{\partial x^{\mu}} d \eta^{\mu}-\frac{\partial H}{\partial \eta^{v}} \frac{\partial \eta^{v}}{\partial x^{\mu}} d x^{\mu} \\
& -\frac{\partial \eta^{\mu}}{\partial x^{\mu}} d \varphi-\sum_{\mu \neq v}\left(\frac{\partial \varphi}{\partial x^{v}} \frac{\partial \eta^{v}}{\partial x^{\mu}} \quad \frac{\partial \varphi}{\partial x^{\mu}} \frac{\partial \eta^{v}}{\partial x^{v}}\right) d x^{\nu}=0
\end{aligned}
$$

(to the last terme the Einstein's convention is not applied). Hence

$$
\begin{gathered}
\frac{\partial \varphi}{\partial x^{\mu}}+\frac{\partial H}{\partial \eta^{\mu}}=0, \quad \frac{\partial \eta^{\mu}}{\partial x^{\mu}}-\frac{\partial H}{\partial \varphi}=0 \\
\frac{\partial H}{\partial \varphi} \frac{\partial \varphi}{\partial x^{\mu}}+\frac{\partial H}{\partial \eta^{v}} \frac{\partial \eta^{v}}{\partial x^{\mu}}+\sum_{\mu \neq v}\left(\frac{\partial \varphi}{\partial x^{v}} \frac{\partial \eta^{v}}{\partial x^{\mu}}-\frac{\partial \varphi}{\partial x^{\mu}} \frac{\partial \eta^{v}}{\partial x^{v}}\right)=0 .
\end{gathered}
$$

It can be shown that here also "energy equations" (6) are consequence of "canonical equations" (5).

If we put e.g. $H\left(x^{\mu}, \varphi, \eta^{\mu}\right)=-\frac{1}{2}\left(\eta_{\mu} \eta^{\mu}+m^{2} \varphi^{2}\right)$, where $\eta_{\mu}=g_{\mu \nu} \eta^{v}, g_{\mu v}$ is Minkowski's metric tensor in space-time, then Eqs. (5) read:

$$
\frac{\partial \varphi}{\partial x^{\mu}}=\eta_{\mu}, \quad \frac{\partial \eta_{\mu}}{\partial x^{\mu}}=-m^{2} \varphi .
$$

It is the theory of Klein-Gordon field:

$$
\left(\square+m^{2}\right) \varphi=0
$$

where $\square=\partial_{\mu} \partial^{\mu}$. 
4. Electrodynamics. We take $W=T^{*}(M)$ where $M$ is space-time. Taking any coordinate chart $\left(x^{\mu}\right)$ in $M$ we have automatically the coordinate chart $\left(x^{\mu}, A_{v}\right)$ in $W$ :

$$
\boldsymbol{A}=A_{\mu} d x^{\mu} \in T^{*}(M) .
$$

The covector $\boldsymbol{A}$ plays role of the electromagnetic potential. The space $W$ plays role of the configuration space. As the phase space $\mathscr{P}$ we take the submanifold in $\bigwedge^{4} T^{*}(W)$ composed of all the 4-covectors of the form:

$$
h d x^{0} \wedge \cdots \wedge d x^{3}+h^{\mu \nu} d x^{0} \wedge \cdots \wedge \underset{\wedge}{\wedge} A_{\mu} \wedge \cdots \wedge d x^{3}
$$

where the following "constraints" are imposed:

$$
h=H\left(x^{\mu}, A_{\mu}, h^{\mu \nu}\right) ; \quad h^{\mu \nu}=-h^{\mu \nu} .
$$

Take $\gamma=d \omega \mid \mathscr{P}=d H \wedge d x^{0} \wedge \cdots \wedge d x^{3}+d h^{\mu v} \wedge d x^{0} \wedge \cdots \wedge \underset{\wedge}{\wedge} A_{\mu} \wedge \cdots \wedge d x^{3}$ where $\omega$ is canonical form in $\bigwedge^{4} T^{*}(W)$.

The space $\mathscr{P}$ is 14 -dimensional with coordinates $\left(x^{\mu}, A_{\mu}, h^{\mu v}\right)$ (only 6 coefficients of antisimetric tensor $h^{\mu v}$ are independent).

The canonical equations can be easily obtained (we leave out the computation):

$$
\begin{aligned}
\partial_{\mu} A_{v}-\partial_{\nu} A_{\mu} & =\frac{\partial H}{\partial h^{\mu \nu}}, \\
\partial_{\nu} h^{\mu \nu} & =\frac{\partial H}{\partial A_{\mu}} .
\end{aligned}
$$

Denoting $\frac{\partial H}{\partial h^{\mu \nu}}=f_{\mu \nu} ; \frac{\partial H}{\partial A_{\mu}}=j^{\mu}$ we see that our equations are nothing but equations of general non-linear electrodynamics (cf. [2, 3]). Specifying the theory to the case of Maxwell electrodynamics with vanishing currents we obtain:

$$
\begin{aligned}
H=\frac{1}{4} h^{\mu v} h_{\mu v} ; \quad f_{\mu \nu} & =\frac{\partial H}{\partial h^{\mu v}}=h_{\mu \nu} ; j^{\mu}=0 \\
f_{\mu v} & =\partial_{\mu} A_{v}-\partial_{v} A_{\mu} \\
\partial_{v} f^{\mu \nu} & =0 .
\end{aligned}
$$

\section{Canonical Fields. Cauchy Surfaces}

If the complete vector field $X$ leaves the form $\gamma$ invariant:

$$
\mathscr{L}_{X} \gamma=0
$$

then the group of diffeomorphisms $\left\{G_{t}^{X}\right\}_{t \in R}$ generated by $X$ carries singular subspaces of $\gamma$ (elements of $\mathscr{A}^{\prime}$ ) without losing this property. 
It means that $\left\{G_{t}^{X}\right\}$ transform states onto states. So $X$ induces a group of transformations $\left\{\mathscr{G}_{t}^{X}\right\}$ in $\mathscr{H}$. If $\mathscr{H}$ has a differentiable structure (which can be often defined by similar methods as in [7] or [9]) this group of transformations defines in $\mathscr{H}$ the vector field $\mathscr{X}$.

Fields satisfying (7) will be called canonical fields. So we have shown

Proposition 1. Every complete canonical field in $\mathscr{P}$ generates a group of transformations and possibly a vector field in $\mathscr{H}$.

Let $X$ be any canonical gauge-field. The group $\left\{\mathscr{G}_{t}^{X}\right\}$ generated by $X$ is trivial (gauge fields are tangent to every state) so the correspondent field $\mathscr{X}$ in $\mathscr{H}$ vanishes.

The following fact is true

Lemma 2. 1. If $X_{1}, X_{2}$ are canonical then $\left[X_{1}, X_{2}\right]$ is canonical, too.

2. If $X$ is canonical and $Y$-canonical gauge field then $[X, Y]$ is canonical gauge field.

Proof. 1. $\mathscr{L}_{\left[X_{1}, X_{2}\right]} \gamma=\left(\mathscr{L}_{X_{1}} \mathscr{L}_{X_{2}}-\mathscr{L}_{X_{2}} \mathscr{L}_{X_{1}}\right) \gamma=0$.

2. $[X, Y]=\lim _{t \rightarrow 0} \frac{1}{t}\left\{\left(G_{t}^{X}\right)_{*} Y-Y\right\}$, where $\left(G_{t}^{X}\right)_{*}$ is the tangent mapping generated by $\left(G_{t}^{X}\right)$. Because $X$ is canonical field, then $\left(G_{t}^{X}\right)_{*} Y$ is also tangent to every state i.e. is a gauge field. Thus $[X, Y]$ is also a gauge field. It follows from 1. that it is canonical.

This result means that the space of canonical fields is a Lie algebra and that canonical gauge fields form its ideal. So the quotient space

$$
\mathscr{Z}^{\prime}=\text { canonical fields/canonical gauge fields }
$$

is a Lie algebra. If we have in $\mathscr{H}$ a differentiable structure then elements of $\mathscr{Z}^{\prime}$ generate vector fields in $\mathscr{H}$. Denote by $\mathscr{Z}$ the set of all thus obtained vector fields in $\mathscr{H}$.

Theorem 1. The correspondence $\mathscr{Z}^{\prime} \rightarrow \mathscr{Z}$ is an isomorphism of Lie algebras

Proof. It is a homomorphism as a consequence of the fact that the correspondence of canonical transformations $G_{t}^{X} \rightarrow \mathscr{G}_{t}^{X}$ is a homomorphism of groups. Now let $\tilde{X} \in \mathscr{Z}^{\prime}$ be in the kernel of this homomorphism. It means that for every representant $X \in \tilde{X}$ and every state $\Omega$ we have

$$
G_{t}^{X}(\Omega)=\Omega .
$$

It means that $X$ is a gauge field.

The elements of $\mathscr{Z}$ will be called canonical fields in $\mathscr{H}$.

Very often we are interested in the minimal set of information which enables to distinguish the concrete state $\Omega \in \mathscr{H}$. In mechanics it suffices 
to know one single point $p \in \Omega$ (the initial position and momentum). In the field theories the field equations are, in general, of hyperbolic character so Cauchy data determine the whole state. In our axiomatic formulation we take the following

Definition. The submanifold $C \subset \mathscr{P}$ is called the Cauchy surface for $(\mathscr{P}, \gamma)$ if $C \cap \Omega$ is $(n-1)$-dimensional for every $\Omega \in \mathscr{H}$ and determines uniquely the state - i.e.

$$
\left(\Omega_{1} \cap C=\Omega_{2} \cap C\right) \Rightarrow\left(\Omega_{1}=\Omega_{2}\right) .
$$

Example. In the field theory $\mathscr{P}$ is often a bundle over space-time $M$. If $\Sigma \subset M$ is a 3-dimensional space-like surface, then the reduction $\mathscr{P}_{\Sigma}$ of $\mathscr{P}$ to $\Sigma$ is (for hyperbolic cases) a Cauchy surface.

Definition. The $(n-1)$-dimensional submanifold $c \subset \mathscr{P}$ is called the initial surface if there exists a unique state $\Omega \in \mathscr{H}$ containing $c(c \subset \Omega)$.

If $C$ is Cauchy surface for $(\mathscr{P}, \gamma)$ then obviously $\Omega \cap C$ is an initial surface for any $\Omega \in \mathscr{H}$.

We do not solve in this paper global problems, thus the needed global properties of our space must be assumed. The most natural way of doing it is to assume that there exists in $\mathscr{P}$ a suitably rich family $\mathscr{C}$ of initial surfaces, which satisfies some axioms. Because these axioms will be used only in several proofs, we shall formulate them in Chapter 11. Now we take the following

Definition. Elements of $\mathscr{C}$ will be called "admissible initial surfaces" (a.i.s.).

Examples. 1. For good hyperbolic field theory the family

$$
\mathscr{C}=\left\{\Omega \cap \mathscr{P}_{\Sigma}: \Omega \in \mathscr{H}, \Sigma \text { is space-like surface }\right\}
$$

satisfies axioms of Chapter 11.

2. In mechanics the set of all points of $\mathscr{P}$ (0-dimensional submanifolds) also satisfies those axioms.

\section{Local Observables. Poisson Bracket}

If $X$ is a canonical field then

$$
(-1)^{n} \mathscr{L}_{X} \gamma=d(X-\gamma)=0 .
$$

It means that (locally) there exists a $(n-1)$ form $\alpha$ for which

$$
d \alpha=X\lrcorner \gamma .
$$

We take the following

Definition. We say that the field $X$ is generated by a local observable if there exists (globally) the $(n-1)$-form $\alpha$ which satisfies conditions: 
a) the cut-off of $\alpha$ to every a.i.s. $c \in \mathscr{C}$ (i.e. $\alpha \mid c$ ) has compact support.

b) $d \alpha=X\lrcorner \gamma$.

Let $\alpha$ be as in the above definition. For every a.i.s. $c \in \mathscr{C}$ put

$$
\langle c, \alpha\rangle=\int_{c} \alpha .
$$

The following is true

Theorem 2. The value of $\langle c, \alpha\rangle$ does not depend on the particular choice of $c \subset \Omega$.

Thus we can denote for $c \subset \Omega$ :

$$
\left\langle\Omega, O_{\alpha}\right\rangle=\langle c, \alpha\rangle \text {. }
$$

Definition. The functional

$$
\mathscr{H} \ni \Omega \rightarrow\left\langle\Omega, O_{\alpha}\right\rangle
$$

will be called a local observable (represented by the form $\alpha$ ). The set of all local observables will be denoted $\mathcal{O}_{0}(\mathscr{P}, \gamma)$ or simply $\mathcal{O}_{0}$.

We shall also use the following notation: $O_{\alpha}=\dot{\alpha}$ (the class of all forms giving the same functional).

Theorem 2 results, practically, from the field equations:

$$
d(\alpha \mid \Omega)=d \alpha|\Omega=(X-\gamma)| \Omega=0 .
$$

It means that every local observable is given by the conservated vector current $\alpha \mid \Omega$ (for detailed proof see Chapter 11).

Remarks. If $\alpha$ represents a local observable then

1. for any $(n-2)$ form $\lambda$ for which $\operatorname{supp}(\lambda \mid c)$ is compact for any a.i.s. the form $\alpha+d \lambda$ represents the same observable because $\langle c, d \lambda\rangle=0$.

2. If $\delta$ is any $(n-1)$-form vanishing on every a.i.s. (i.e. $\delta \mid c=0$ for $c \in \mathscr{C}$ ) then $\alpha+\delta$ represents the same observable.

For illustrate the Remark 2 take $\delta=(Y \wedge X) \downarrow \gamma$ where $Y$ is a gauge field and $X-$ any vector field in $\mathscr{P}$. If $u$ is any $(n-1)$ vector tangent to some a.i.s. at the point $p \in \mathscr{P}$, then $u \wedge Y_{p}$ is an $n$-vector tangent to a state passing by $p$. Then

$$
\left.\left\langle u,\left(Y_{p} \wedge X_{p}\right)-\gamma_{p}\right\rangle=\left\langle u \wedge Y_{p}, X_{p}\right\lrcorner \gamma_{p}\right\rangle=0 .
$$

The following fundamental fact is true:

Theorem 3. If $X_{1}$ and $X_{2}$ are generated by the same observable $O_{x} \in \mathcal{O}_{0}$ then they belong to the same class modulo gauge fields. It means that an observable generates a unique element of $\mathscr{Z}^{\prime}$, i.e. a unique canonical field on $\mathscr{H}$ (for the proof see Chapter 11). 
If $O_{1}, O_{2} \in \mathcal{O}_{0}$ are local observables generating canonical fields $X_{i}$ and if $\alpha_{i}$ are their representants, we put

$$
\begin{aligned}
\left\{\alpha_{1}, \alpha_{2}\right\} & \left.\left.=\mathscr{L}_{X_{1}} \alpha_{2}=(-1)^{n-1}\left\{X_{1}\right\lrcorner d \alpha_{2}-d\left(X_{1}\right\lrcorner \alpha_{2}\right)\right\} \\
& \left.\left.=(-1)^{n-1}\left(X_{1} \wedge X_{2}\right)\right\lrcorner \gamma+(-1)^{n} d\left(X_{1}\right\lrcorner \alpha_{2}\right) .
\end{aligned}
$$

The form $\left\{\alpha_{1}, \alpha_{2}\right\}$ has obviously compact support on every a.i.s. Moreover we have following

Lemma 3. $\left.d\left\{\alpha_{1}, \alpha_{2}\right\}=\left[X_{1}, X_{2}\right]\right\lrcorner \gamma$.

Proof. $\left.\left.d\left\{\alpha_{1}, \alpha_{2}\right\}=d\left(\mathscr{L}_{X_{1}} \alpha_{2}\right)=\mathscr{L}_{X_{1}}\left(d \alpha_{2}\right)=\mathscr{L}_{X_{1}}\left(X_{2}\right\lrcorner \gamma\right)=\left[X_{1}, X_{2}\right]\right\lrcorner \gamma$ $\left.\left.+X_{2}\right\lrcorner\left(\mathscr{L}_{X_{1}} \gamma\right)=\left[X_{1}, X_{2}\right]\right\lrcorner \gamma$.

The field $\left[X_{1}, X_{2}\right]$ is also canonical. Thus $\left\{\alpha_{1}, \alpha_{2}\right\}$ represents an observable $\left\{\alpha_{1}, \alpha_{2}\right\} \in \mathcal{O}_{0}$. This observable does not depend on particular choice of $\alpha_{1}$ and $\alpha_{2}$ : if we take another representants so only the second terme of (8) will change. It follows from Remark 1 that $\left\{\alpha_{1}, \alpha_{2}\right\}^{\prime}$ do not change. The observable $\left\{\alpha_{1}, \alpha_{2}\right\}^{\prime}$ is also independent on particular choice of fields generated by observables $O_{i}$. It can be concluded from Theorem 3 and Remark 2: if we add to $X_{i}$ gauge fields $Y_{i}$ the only result will be adding to $\left\{\alpha_{1}, \alpha_{2}\right\}$ terms vanishing on every a.i.s.

Thus $\left\{\alpha_{1}, \alpha_{2}\right\}$ depends only of $O_{1}$ and $O_{2}$. We shall denote

$$
\left\{\alpha_{1}, \alpha_{2}\right\}=\left\{O_{1}, O_{2}\right\} \text {. }
$$

Definition. The observable $\left\{O_{1}, O_{2}\right\}$ will be called the Poisson bracket of $O_{1}$ and $O_{2}$.

From Lemma 3 we have an immediate

Corollary. If $O_{1}$ generates $X_{1}$ and $O_{2}$ generates $X_{2}$ then $\left\{O_{1}, O_{2}\right\}$ generates $\left[X_{1}, X_{2}\right]$.

To collect the above facts we write:

$$
\left.\left\{O_{1}, O_{2}\right\}=\left(\mathscr{L}_{X_{1}} \alpha_{2}\right)^{\cdot}=(-1)^{n-1}\left\{\left(X_{1} \wedge X_{2}\right)\right\lrcorner \gamma\right\}^{\cdot} .
$$

We could say that $\left\{O_{1}, O_{2}\right\}$ gives the change of an observable $O_{2}$ when we move on trajectories (in $\mathscr{H}$ ) generated by $O_{1}$.

The set $\mathcal{O}_{0}$ has obviously linear structure. The Poisson bracket is bilinear mapping. Moreover the following is true:

Proposition 2. The space $\left(\mathcal{O}_{0},\{\}\right)$ is a Lie algebra, i.e.:

1. $\left\{O_{1}, O_{2}\right\}=-\left\{O_{2}, O_{1}\right\}$

2. $\left\{O_{1},\left\{O_{2}, O_{3}\right\}\right\}+\left\{O_{2},\left\{O_{3}, O_{1}\right\}\right\}+\left\{O_{3},\left\{O_{1}, O_{2}\right\}\right\}=0$ (zero observable).

Proof. 1. is obvious from the expression $\left.(-1)^{n-1}\left\{\left(X_{1} \wedge X_{2}\right)\right\lrcorner \gamma\right\}$ : 
2. $\left\{\alpha_{1},\left\{\alpha_{2}, \alpha_{3}\right\}\right\}^{\cdot}+\left\{\alpha_{2},\left\{\alpha_{3}, \alpha_{1}\right\}\right\}^{\cdot}+\left\{\alpha_{3},\left\{\alpha_{1}, \alpha_{2}\right\}\right\}^{\circ}$

$$
\begin{aligned}
& =\left\{\alpha_{1},\left\{\alpha_{2}, \alpha_{3}\right\}\right\}^{\cdot}-\left\{\alpha_{2},\left\{\alpha_{1}, \alpha_{3}\right\}\right\}^{\cdot}-\left\{\left\{\alpha_{1}, \alpha_{2}\right\}, \alpha_{3}\right\}^{\cdot} \\
& =\left\{\mathscr{L}_{X_{1}} \mathscr{L}_{X_{2}} \alpha_{3}\right\}^{\cdot}-\left\{\mathscr{L}_{X_{2}} \mathscr{L}_{X_{1}} \alpha_{3}\right\}^{\cdot}-\left\{\mathscr{L}_{\left[X_{1}, X_{2}\right]} \alpha_{3}\right\}^{\cdot} \\
& =\left\{\left[\left(\mathscr{L}_{X_{1}} \mathscr{L}_{X_{2}}-\mathscr{L}_{X_{2}} \mathscr{L}_{X_{1}}\right)-\mathscr{L}_{\left[X_{1}, X_{2}\right]}\right] \alpha_{3}\right\}^{\cdot}=0 .
\end{aligned}
$$

The mapping $\mathcal{O}_{0} \ni O_{\alpha} \rightarrow \mathscr{X}_{\boldsymbol{O}_{\alpha}} \in \mathscr{Z}$, where $\mathscr{X}_{\boldsymbol{O}_{\alpha}}$ is canonical field in $\mathscr{H}$ generated by the observable $O_{\alpha}$, is a homomorphism of Lie algebras. It follows immediately from Lemma 3:

$$
X_{\left\{O_{1}, O_{2}\right\}}=\left[X_{O_{1}}, X_{O_{2}}\right] .
$$

It is worth noticing that the kernel of this mapping is composed of constant observables:

Theorem 4. If $\mathscr{X}_{O_{\alpha}} \in \mathscr{Z}$ is equal zero then there exists a number: $a \in R$ such that

$$
\left\langle\Omega, O_{\alpha}\right\rangle \equiv a
$$

for every $\Omega \in \mathscr{H}$ (proof in Chapter 11).

\section{Current Algebra. Noether Theorem}

In theories of Lagrangean origine $\gamma$ is always an exterior derivative of the Lagrangean density $\omega$ (cf. [6]): $\gamma=d \omega$. Vector field $X$ which leaves Lagrangean invariant $\left(\mathscr{L}_{X} \omega=0\right)$ is called the symmetry field. In this language the Noether theorem reads:

Theorem (Noether). Every symmetry field generates on every state the conservated current.

Proof. Take $\alpha=X-\omega$. Then for any state $\Omega$ we have:

$$
\left.\left.d \alpha=d(X\lrcorner \omega)=(-1)^{n+1} \mathscr{L}_{X} \omega+X\right\lrcorner d \omega=X\right\lrcorner \gamma .
$$

Thus $d \alpha \mid \Omega=(X-\gamma) \Omega=0$.

If $\alpha$ had compact support on every a.i.s. then the formula $d \alpha=X-\gamma$ could be read: $X$ is generated by local observable. But generally it is not true. Such forms represent "global observables" like energy, momentum, electric charge etc.

Definition. By "current" or "global observable" we mean the functional $\langle c, X\lrcorner \omega\rangle$, where $X$ is a symmetry field. Such a functional is, in general, well defined only on a subspace of $\mathscr{H}$ and not on the whole space (cf. Chapter 8). The space of all currents will be denoted $\mathscr{J}$.

The same construction can be made for symmetry fields and global observables as in the precedent chapter for canonical fields and local 
observables. Note, for instance, that symmetry fields are canonical. They form a Lie algebra:

$$
\mathscr{L}_{\left[X_{1}, X_{2}\right]} \omega=\left(\mathscr{L}_{X_{1}} \mathscr{L}_{X_{2}}-\mathscr{L}_{X_{1}} \mathscr{L}_{X_{2}}\right) \omega=0 .
$$

For two currents represented by forms $\alpha, \beta$ and generated by symmetry fields $X_{\alpha}, X_{\beta}$ we put

$$
\begin{aligned}
\left.\{\alpha, \beta\}=\mathscr{L}_{X_{\alpha}} \beta=\mathscr{L}_{X_{\alpha}}\left(X_{\beta}\right\lrcorner \omega\right) & \left.\left.=\left[X_{\alpha}, X_{\beta}\right]\right\lrcorner \omega+X_{\beta}\right\lrcorner\left(\mathscr{L}_{X_{\alpha}} \omega\right) \\
& \left.=\left[X_{\alpha}, X_{\beta}\right]\right\lrcorner \omega
\end{aligned}
$$

It means that $\mathscr{J}$ is a Lie algebra homomorphic with the algebra of symmetry fields.

Our definition of a current bases on the fact that we have chosen a concrete primitive form $\omega$ for $\gamma$. It is an additional structure in our phase space $(\mathscr{P}, \gamma)$ and enables us to take a concrete representant $X\lrcorner \omega$ instead of a whole class of forms.

When $X$ is a symmetry field and $O_{\alpha} \in \mathcal{O}_{0}$ a local observable, then $\mathscr{L}_{X} \alpha$ has compact support on every a.i.s., for any representant $\alpha$ of $O_{x}$. It enables us to define full algebra of observables

$$
\mathcal{O}=\mathscr{J} \oplus \mathcal{O}_{0}
$$

putting $\left\{\beta, O_{\alpha}\right\}=\left\{\mathscr{L}_{X} \alpha\right\}^{\circ} \in \mathcal{O}_{0}$, where $\beta \in \mathscr{J}$ is generated by $X . \mathscr{J}$ and $\mathscr{O}_{0}$ are subalgebras of $\mathcal{O}$ and $\mathcal{O}_{0}$ is even its ideal.

\section{Examples of Observables. Observable-valued Distributions}

1. In mechanics $(n=1)$ observables are 0 -forms, i.e. functions. The condition $d \alpha \mid \Omega=0$ means that they are constant on whole states. They are therefore constant of motion.

Let us study the condition for $\frac{\partial}{\partial t}$ being the symmetry field:

$$
\begin{aligned}
\mathscr{L}_{\frac{\partial}{\partial t} \omega} & \left.\left.=-d\left(\frac{\partial}{\partial t}\right\lrcorner p_{\mu} d x^{\mu}\right)+\frac{\partial}{\partial t}\right\lrcorner\left(d p_{\mu} \wedge d x^{\mu}\right) \\
& =-d p_{0}-\frac{\partial p_{0}}{\partial t} d t+d p_{0}=0 .
\end{aligned}
$$

It means that $\frac{\partial}{\partial t}$ is a symmetry field if and only if the Hamiltonian does not depend on time.

2. Take the Klein-Gordon theory:

$$
\begin{aligned}
& \omega=-\frac{1}{2}\left(\eta_{\mu} \eta^{\mu}+m^{2} \varphi^{2}\right) d x^{0} \wedge \cdots \wedge d x^{3}+\eta^{\mu} d x^{0} \wedge \cdots \wedge \underset{\hat{\mu}}{d \varphi} \wedge \cdots \wedge d x^{3}, \\
& \gamma=-\left(\eta_{\mu} d \eta^{\mu}+m^{2} \varphi d \varphi\right) \wedge d x^{0} \wedge \cdots \wedge d x^{3}+d \eta^{\mu} \wedge d x^{0} \wedge \cdots \wedge \underset{\hat{\mu}}{d \varphi} \wedge \wedge \wedge d x^{3} .
\end{aligned}
$$


It is easy to compute (cf. Appendix) that the Lie algebra of Poincare group is an algebra of symmetry fields of our theory. So the current algebra is 10-dimensional. For instance the energy $E$ generated by the field $\frac{\partial}{\partial x^{0}}$ is represented by the 3 -form:

$$
\begin{aligned}
\alpha= & \left.\frac{\partial}{\partial x^{0}}\right\lrcorner \omega=\frac{1}{2}\left(\eta_{\mu} \eta^{\mu}+m^{2} \varphi^{2}\right) d x^{1} \wedge d x^{2} \wedge d x^{3} \\
& +\sum_{i=1}^{3} \eta^{i} d x^{1} \wedge \cdots \wedge \underset{i}{d} \varphi \wedge \cdots \wedge d x^{3} .
\end{aligned}
$$

Take as a Cauchy surface the reduction of $\mathscr{P}$ to the space-like surface $\Sigma$ given by equation $x^{0}=$ const. Using the canonical equations $\eta_{\mu}=\frac{\partial \varphi}{\partial x^{\mu}}$ we obtain for any state $\Omega \in \mathscr{H}$ :

$$
\begin{aligned}
\langle\Omega, E\rangle=\int_{\Omega \cap C} \alpha & =\int_{\Sigma}\left\{\frac{1}{2}\left(\eta_{\mu} \eta^{\mu}+m^{2} \varphi^{2}\right)+\sum_{i=1}^{3} \eta^{i} \eta_{i}\right\} d x^{1} \wedge d x^{2} \wedge d x^{3} \\
& =\frac{1}{2} \int_{\Sigma}\left(\sum_{\mu=0}^{4}\left(\frac{\partial \varphi}{\partial x^{\mu}}\right)^{2}+m^{2} \varphi^{2}\right) d^{3} \boldsymbol{x} .
\end{aligned}
$$

We see that it is well defined (finite) only for states sufficiently rapidly vanishing at infinity.

In the case of wave-equation $(m=0)$ also $\frac{\partial}{\partial \varphi}$ is a symmetry field. The correspondent current is:

$$
\begin{gathered}
\left.\beta=\frac{\partial}{\partial \varphi}\right\lrcorner \omega=\Sigma(-1)^{\mu} \eta^{\mu} d x^{0} \wedge \cdots \hat{\mu}_{\mu} \cdots \wedge d x^{3}, \\
\langle\Omega, \beta\rangle=\int_{\Sigma} \eta^{0} d x^{1} \wedge d x^{2} \wedge d x^{3}=\int_{\Sigma} \frac{\partial \varphi}{\partial x^{0}} d^{3} \boldsymbol{x} .
\end{gathered}
$$

Now we pass to local observables. Let $f: M \rightarrow R$ be any solution of Klein-Gordon equation: $\left(\square+m^{2}\right) f=0$. Take the field

$$
X_{f}=\partial^{\mu} f \cdot \frac{\partial}{\partial \eta^{\mu}}+f \frac{\partial}{\partial \varphi} .
$$

It is a canonical field:

$$
\begin{aligned}
\left.X_{f}\right\lrcorner \gamma= & \left(-\eta_{\mu} \cdot \partial^{\mu} f\right) d x^{0} \wedge \cdots \wedge d x^{3}+\partial^{\mu} f \cdot d x^{0} \wedge \cdots \wedge \underset{\mu}{d \varphi} \wedge \cdots \wedge d x^{3} \\
& -m^{2} f \cdot \varphi \cdot d x^{0} \wedge \cdots \wedge d x^{3}-(-1)^{\mu} f \cdot d \eta^{\mu} \wedge d x^{0} \wedge \cdots{ }_{\mu} \cdots \wedge d x^{3}
\end{aligned}
$$


and one can easily obtain $d\left(X_{f}-\gamma\right)=0$. Take the 3 -form

$$
\alpha_{f}=\Sigma(-1)^{\mu}\left(\varphi \cdot \partial^{\mu} f-f \cdot \eta^{\mu}\right) d x^{0} \wedge \cdots \hat{\mu}_{\mu}^{\cdots \wedge d x^{3}} .
$$

The reader can check that $\left.d \alpha_{f}=X_{f}\right\lrcorner \gamma$.

If $f$ has spatially-bounded support, then so does $\alpha_{f}$. In this case $\alpha_{f}$ represents a local observable which we shall denote $O_{f}$. Integrating over the surface $t=$ const we obtain

$$
\left\langle\Omega, O_{f}\right\rangle=\int_{\Sigma}\left(\varphi \cdot \frac{\partial f}{\partial x^{0}}-f \frac{\partial \varphi}{\partial x^{0}}\right) d^{3} \boldsymbol{x}=\int_{\Sigma}\left(f \stackrel{\leftrightarrow}{\partial_{\mu}} \varphi\right) d \sigma^{\mu}
$$

(the last expression being valid for any space-like surface $\Sigma$ ). Taking in particular $f$ satisfying the following Cauchy conditions:

$$
f\left|\Sigma=0 \quad \frac{\partial f}{\partial x^{0}}\right| \Sigma=\psi
$$

we define the observable:

$$
\langle\Omega, O(\Sigma, \varphi, \psi)\rangle=\int_{\Sigma} \varphi \cdot \psi d^{3} \boldsymbol{x} .
$$

Taking $f\left|\Sigma=-\chi, \frac{\partial f}{\partial x^{0}}\right| \Sigma=0$ we define

$$
\left\langle\Omega, O\left(\Sigma, \eta_{0}, \chi\right)\right\rangle=\int_{\Sigma} \frac{\partial \varphi}{\partial x^{0}} \cdot \chi d^{3} \boldsymbol{x} .
$$

The above observables can be called "the value of $\varphi$ at the time $x^{0}$, smeared with test function $\psi$ " and "the value of $\eta_{0}$ at the time $x^{0}$, smeared with $\chi$ ". If the functions $\psi, \chi$ are very close to Dirac delta: $\delta\left(\boldsymbol{x}-\boldsymbol{x}_{0}\right)$ then the value of (10) and (11) is very close to $\varphi\left(x^{0}, \boldsymbol{x}_{0}\right)$ and $\eta_{0}\left(x^{0}, \boldsymbol{x}_{0}\right)$. Thus similarly as in quantum field theory only "smeared" fields are observables. To simplify the notation we can introduce the observable-valued distributions:

$$
\begin{aligned}
& \hat{\varphi}: \mathscr{D}(\Sigma) \rightarrow \mathcal{O}, \\
& \hat{\eta}_{0}: \mathscr{D}(\Sigma) \rightarrow \mathcal{O} .
\end{aligned}
$$

$\left(\mathscr{D}(\Sigma)=C_{0}^{\infty}(\Sigma)\right.$ with the usual topology) setting:

$$
\begin{aligned}
& \int \hat{\varphi}(\boldsymbol{x}) \psi(\boldsymbol{x}) d^{3} \boldsymbol{x}:=\hat{\varphi}(\psi)=O(\Sigma, \varphi, \psi), \\
& \int \hat{\eta}_{0}(\boldsymbol{x}) \chi(\boldsymbol{x}) d^{3} \boldsymbol{x}:=\hat{\eta}_{0}(\chi)=O\left(\Sigma, \eta_{0}, \chi\right) .
\end{aligned}
$$

The Poisson bracket of our observables can be easily computed:

$$
\begin{gathered}
\left\{O_{f}, O_{g}\right\}=\left(\left(X_{f} \wedge X_{g}\right)-\gamma\right)^{\cdot}=\Sigma(-1)^{\mu}\left(g \cdot \partial^{\mu} f-f \cdot \partial^{\mu} g\right) d x^{0} \wedge \cdots \hat{\mu}_{\mu} \cdots \wedge d x^{3}, \\
\left\langle\Omega,\left\{O_{f}, O_{g}\right\}\right\rangle=\int_{\Sigma} g \overleftrightarrow{\partial_{\mu}} f d \sigma^{\mu} .
\end{gathered}
$$


In particular:

$$
\begin{gathered}
\left\{O\left(\Sigma, \varphi, \psi_{1}\right), O\left(\Sigma, \varphi, \psi_{2}\right)\right\}=0=\left\{O\left(\Sigma, \eta_{0}, \chi_{1}\right), O\left(\Sigma, \eta_{0}, \chi_{2}\right)\right\}, \\
\left\{O(\Sigma, \varphi, \psi), O\left(\Sigma, \eta_{0}, \chi\right)\right\}=\int_{\Sigma} \psi \cdot \chi d^{3} x .
\end{gathered}
$$

If we strip these formulae of test functions $\psi, \chi$ we obtain at last:

$$
\begin{gathered}
\left\{\hat{\varphi}\left(x^{0}, \boldsymbol{x}\right), \hat{\varphi}\left(x^{0}, \boldsymbol{y}\right)\right\}=0=\left\{\hat{\eta}_{0}\left(x^{0}, \boldsymbol{x}\right), \hat{\eta}_{0}\left(x^{0}, \boldsymbol{y}\right)\right\}, \\
\left\{\hat{\varphi}\left(x^{0}, \boldsymbol{x}\right), \hat{\eta}_{0}\left(x^{0}, \boldsymbol{y}\right)\right\}=\delta(\boldsymbol{x}-\boldsymbol{y}) .
\end{gathered}
$$

3. The electrodynamics with potentials is not a hyperbolic theory. But if we replace Cauchy-surfaces by space-like surfaces we can define observables in the same manner as in Chapter 6 and 7. Take e.g. Maxwell electrodynamics with vanishing electric current:

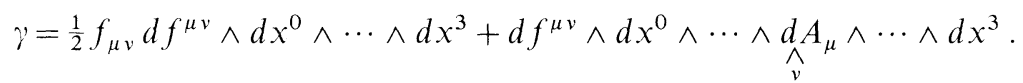

Let $g_{\mu}$ be any solution of canonical equations: $\partial^{\mu}\left(\partial_{\mu} g_{v}-\partial_{v} g_{\mu}\right)=0$. Denote $h_{\mu \nu}=\partial_{\mu} g_{v}-\partial_{v} g_{\mu}$. Take the vector field

$$
X_{g}=g_{\mu} \frac{\partial}{\partial A_{\mu}}+h^{\mu v} \frac{\partial}{\partial f^{\mu v}} .
$$

One can easily check that $X_{g}$ is canonical field. The corresponding observable can be represented by

$$
\alpha_{g}=\Sigma(-1)^{v}\left(A_{\mu} h^{\mu v}-g_{\mu} f^{\mu v}\right) d x^{0} \wedge \cdots \hat{\nu}_{v}^{\cdots \wedge d x^{3} .}
$$

For $\Sigma$ given by equality $t=$ const take $g_{i}\left|\Sigma=-\delta_{i}^{k} \cdot \psi ; h_{0 i}\right| \Sigma=0$. We obtain the observable:

$$
\left\langle\Omega, O\left(\Sigma, E^{k}, \psi\right)\right\rangle=\int_{\Sigma} f^{k 0} \cdot \psi d^{3} \boldsymbol{x}=\int_{\Sigma} E^{k} \cdot \psi d^{3} \boldsymbol{x},
$$

i.e. the $k$ th component of electric field $\left(E^{k}\right)$ smeared with the function $\psi$. Taking $g_{i}\left|\Sigma=0, g_{0}\right| \Sigma$-arbitrary we obtain the integral

$$
\int_{\Sigma} A_{\mu} h^{\mu 0} d^{3} \boldsymbol{x} .
$$

One might suppose that it is value of potential, smeared with $h^{\mu 0}$. But functions $h^{\mu 0}$ are not arbitrary. They satisfy Maxwell equations which read:

$$
\partial_{\mu} h^{\mu 0}=\sum_{i=1}^{3} \frac{\partial}{\partial x^{i}} h^{i 0}=0 .
$$


It means that the vector field $\left(h^{i 0}\right)$ on $\Sigma$ is a rotation:

$$
h^{i 0}=2 \varepsilon^{i j k} \partial_{j} \chi_{k} .
$$

If the functions $\chi_{k}$ have compact supports then integrating by parts we transform the integral (12) onto the following expression:

$$
\begin{aligned}
2 \int_{\Sigma} \varepsilon^{i j k}\left(\partial_{j} \chi_{k}\right) A_{i} d^{3} \boldsymbol{x} & =-2 \int_{\Sigma} \varepsilon^{i j k} \chi_{k}\left(\partial_{j} A_{i}\right) d^{3} \boldsymbol{x} \\
& =\int_{\Sigma} \chi_{k} \varepsilon^{k j i} f_{j i} d^{3} \boldsymbol{x}=\int_{\Sigma} \chi_{k} B^{k} d^{3} \boldsymbol{x},
\end{aligned}
$$

where $B^{k}$ is $k$ th component of magnetic vector. Taking $\chi_{k}=\delta_{k}^{p} \cdot \chi$ we obtain the observables:

$$
\left\langle\Omega, O\left(\Sigma, B^{p}, \chi\right)\right\rangle=\int_{\Sigma} B^{p} \cdot \chi d^{3} x .
$$

The Poisson bracket can be easily obtained:

where

$$
\left\{\alpha_{g}, \alpha_{\tilde{g}}\right\}=\Sigma\left(h^{\mu v} \cdot \tilde{g}_{\mu}-\tilde{h}^{\mu v} g_{v}\right)(-1)^{v} d x^{0} \wedge \cdots \hat{\imath}_{v} \cdots \wedge d x^{3} .
$$

$$
\tilde{h}_{\mu \nu}=\partial_{\mu} \tilde{g}_{\nu}-\partial_{\nu} \tilde{g}_{\mu} .
$$

Using this formula and taking $h^{i 0}\left|\Sigma=2 \delta_{k}^{p} \cdot \varepsilon^{i j k} \partial_{j} \chi=2 \varepsilon^{i j p} \partial_{j} \chi, g_{i}\right| \Sigma=0$, $\tilde{h}^{i 0}\left|\Sigma=0, \tilde{g}_{i}\right| \Sigma=-\delta_{i}^{k} \cdot \psi$ we have

$$
\left\langle\Omega,\left\{O\left(\Sigma, B^{p}, \chi\right), O\left(\Sigma, E^{k}, \psi\right)\right\}\right\rangle=-\int_{\Sigma} \varepsilon^{p k j}\left(\partial_{j} \chi\right) \cdot \psi d^{3} \boldsymbol{x} .
$$

Using observable valued distributions $\hat{E}^{k}(\boldsymbol{x}), \hat{B}^{p}(\boldsymbol{x})$ we can write (13) as:

$$
\left\{\hat{B}^{p}\left(x^{0}, \boldsymbol{x}\right), \hat{E}^{k}\left(x^{0}, \boldsymbol{y}\right)\right\}=-\varepsilon^{p k j} \partial_{j} \delta(\boldsymbol{x}-\boldsymbol{y}) .
$$

In the same manner we obtain

$$
\left\{\hat{E}^{i}\left(x^{0}, \boldsymbol{x}\right), \hat{E}^{j}\left(x^{0}, \boldsymbol{y}\right)\right\}=0=\left\{\hat{B}^{i}\left(x^{0}, \boldsymbol{x}\right), \hat{B}^{j}\left(x^{0}, \boldsymbol{y}\right)\right\} .
$$

It is complete set of equal-time Poisson brackets for field strengths (cf. [2]).

\section{Dual Cauchy's Problem. Difficulties in non-linear Theories}

Let $C$ be a Cauchy surface in $\mathscr{P}$. The Cauchy's problem consists in finding the state $\Omega$ when we know $C \cap \Omega$.

Now let be given an $(n-1)$-differential form $\alpha$ on $C$. For any state $\Omega \in \mathscr{H}$ we can define the quantity

$$
\langle\Omega, \alpha\rangle:=\int_{\Omega \cap C} x .
$$


We obtain in this manner a functional on the space $\mathscr{H}$. The problem arises: "for which forms $\alpha$ this functional coincides with an observable?". The problem of finding such an observable when we know $\alpha$ can be called the dual Cauchy's problem.

In non linear theories the dual Cauchy's problem is very often, unfortunately, unsolvable because local observables do not exist. As it will be shown in the Appendix (for the theory of self-interacting scalar field $\left.\left(\square+m^{2}\right) \varphi=\lambda \varphi^{n}\right)$ only symmetry fields are canonical ones. So the observable algebra consists only of global observables. Similar situation we meet in another non-linear theories. It may lead to the following conclusions:

1. Either the solution suggested by [10] (the introduction of higherorder currents) will be satisfactory - in this case very useful suggestions about quantization of non-linear theories can be obtained

2. or there is no satisfactory solution. It can be closely connected with difficulties of non-linear quantization.

In any case it seems that the further investigations of canonical structure of classical theories can give a deep insight into problems of quantization.

\section{Concluding Remarks}

If we leave aside the geometrical beauty, an explicit relativistic invariance (there is no necessity of distinguishing the space-like surface for the definition of Poisson brackets) and other such advantages, our theory can be treated as a method of obtaining, for a given classical theory, the startpoint for quantizing it, namely the triplet $(\mathscr{H}, \mathcal{O}, \mathscr{Z})$. Similar triplet is obtained in usual, infinite-dimensional formulations (cf. the beautiful theory of Segal [14]). The difference between those formulations and the present one is that our algebras $\mathcal{O}$ and $\mathscr{Z}$ are much less. Here observables are not arbitrary functionals on $\mathscr{H}$ but very special ones, which are integrals of very special differential forms.

Such reduction of the space of observables enables us to formulate the theory without functional derivatives (which lead to well known difficulties). Furthermore such reduction is very convenient in quantization when, as it is known, only some subalgebra of usually obtained observable algebra is represented directly in Hilbert space. The question "which subalgebra is such a base of quantization" does not exist in our formulation: In Klein-Gordon theory $\mathcal{O}$ contains the Poincaré-group generators and smeared fields only. In electrodynamics - Poincarégroup generators and smeared electric and magnetic fields only (no potentials!). It seems that our algebra is itself an adequate observable algebra for quantization. 
Algebra $\mathcal{O}$ has moreover a very nice property that it has no commutative structure (in standard formulations observables, as functionals, can be multiplied. In our theory such multiplication has, in general, no sense). So there are no difficulties with a "good order of operators" during quantization.

It is worth noticing that observables are represented by classes of differential forms and are not forms themselves. Thus the whole information included in the observable is its value on states: $\langle\Omega, O\rangle$. This point of view is implied by the mathematical structure of the theory: the Poisson bracket for forms $\{\alpha, \beta\}$ has not the demanded properties (cf. Proposition 2). So there is no difference between "canonical" and "symmetric" energy-momentum tensor (as long as it does not enter-into dynamics, as in General Relativity). Only energy, momentum, electric charge etc. are observables and not their densities.

\section{1. $\mathscr{C}$-axiomatics. Proofs}

We take the following system of axioms which must be satisfied by our family $\mathscr{C}$ of a.i.s.:

1. For every $p \in \mathscr{P}$ and $\Omega$ passing by $p$ there exists $c \in \mathscr{C}$ such that $p \in \mathcal{C} \subset \Omega$.

2. If $(n-1)$-dimensional submanifold $c \subset \mathscr{P}$ has the property that for any $p \in c$ there exists a $c_{p} \in \mathscr{C}$ such that $c \cap c_{p}$ is a neighbourhood of $p$ (in $c$ ) then $c \in \mathscr{C}$.

3. If $c_{1}, c_{2} \in \mathscr{C}$ and $K_{i} \subset c_{i}$ are compact sets, then there exists $c \in \mathscr{C}$ such that $K_{1} \subset\left[c_{1}-\left(c \cap c_{1}\right)\right], K_{2} \subset\left(c \cap c_{2}\right)$ and that $\left[c_{1}-\left(c \cap c_{1}\right)\right]$ is relatively compact in $c_{1}$.

4. If $c_{1}, c_{2}$ lie in the same state $\Omega$ then there exists such a $c \in \mathscr{C}$ satisfying (3) which lie also in $\Omega$.

5. Each two $c_{0}, c_{1} \in \mathscr{C}$ can be joined by a 1-parameter smooth curve $c_{t}$, $t \in[0,1]$ in the sense of $[10]$, i.e. there exists such a complete vector field $X$ that $G_{1}^{X}\left(c_{0}\right)=c_{1}, c_{t}=G_{t}^{X}\left(c_{0}\right) \in \mathscr{C}$.

6. If $c_{0}, c_{1}$ lie in the same state $\Omega$, than the curve $c_{t}$ can be taken in a manner that every $c_{t}$ lies also in $\Omega$.

7. Take any $c \in \mathscr{C}$ and $\Omega \in \mathscr{H}$ containing $c$. For any vector field $X$ tangent to $\Omega$ and having compact support there exists $\varepsilon>0$ such that $G_{t}^{X}(c) \in \mathscr{C}$ for $|t|<\varepsilon$.

8. For every $c \in \mathscr{C}, p \in c, Y_{p} \in T_{p}(\mathscr{P})$ and a neighbourhood $A \subset \mathscr{P}$ of $p$ there exist the vector field $Y$ equal $Y_{p}$ at $p$, whose compact support is contained in $A$ and $\varepsilon>0$, such that $G_{t}^{Y}(c) \in \mathscr{C}$ for $|t|<\varepsilon$.

It is worth noticing that e.g. the set of all initial surfaces in the field theory does not satisfy the axiom (2). 
Before proofs of Theorems 2 and 4 let us note the following fact:

Lemma 4. Let the $(n-1)$-form $\alpha$ have the property that $\alpha \mid c$ has compact support for any $c \in \mathscr{C}$. Then for any $c_{1}, c_{2} \in \mathscr{C}$ there exists such $c \in \mathscr{C}$ that $\left[c_{1}-\left(c \cap c_{1}\right)\right]$ is relatively compact and:

$$
\begin{gathered}
\operatorname{supp}(\alpha \mid c)=\operatorname{supp}\left(\alpha \mid c_{2}\right) \subset\left(c \cap c_{2}\right), \\
\operatorname{supp}\left(\alpha \mid c_{1}\right) \subset\left[c_{1}-\left(c \cap c_{1}\right)\right] .
\end{gathered}
$$

Proof. Take $K_{1}=\operatorname{supp}\left(\alpha \mid c_{1}\right), K_{2}=\operatorname{supp}\left(\alpha \mid c_{2}\right)$. Using the third axiom we can take the a.i.s. $d^{1} \in \mathscr{C}$, such that

$$
\begin{gathered}
\operatorname{supp}\left(\alpha \mid c_{2}\right) \subset\left(d^{1} \cap c_{2}\right), \\
\operatorname{supp}\left(\alpha \mid c_{1}\right) \subset\left[c_{1}-\left(d^{1} \cap c_{1}\right)\right] \text {-relatively compact . }
\end{gathered}
$$

If $\operatorname{supp}\left(\alpha \mid c_{2}\right) \neq \operatorname{supp}\left(\alpha \mid d^{1}\right)$ we can take any compact (in $c_{1}$ ) neighbourhood of $\left[c_{1}-\left(d^{1} \cap c_{1}\right)\right]$ as $K_{1}^{1}$ and any compact neighbourhood (in $\left.c_{2}\right)$ of $c_{2} \cap d^{1}$ as $K_{2}^{1}$. There exist $d^{2} \in \mathscr{C}$ such that

$$
K_{1}^{1} \subset\left(d^{2} \cap c_{1}\right) ; K_{2}^{1} \subset\left[c_{2}-\left(d^{2} \cap c_{2}\right)\right] \text {-relatively compact . }
$$

Now we take any compact neighbourhood (in $c_{1}$ ) of $d^{2} \cap c_{1}$ as $K_{1}^{2}$ and any compact neighbourhood (in $\left.c_{2}\right)$ of $\left[c_{2}-\left(d^{2} \cap c_{2}\right)\right]$ as $K_{2}^{2}$. There exists $d^{3} \in \mathscr{C}$ such that

$$
K_{2}^{2} \subset\left(d^{3} \cap c_{2}\right) ; K_{1}^{2} \subset\left[c_{1}-\left(d^{3} \cap c_{1}\right)\right] \text {-relatively compact . }
$$

Using this method we can construct the sequence $d^{1}, d^{2}, \ldots$ of a.i.s. and $K_{1}^{s} \nearrow c_{1}, K_{2}^{s} \nearrow c_{2}$ as $s \rightarrow \infty$ :

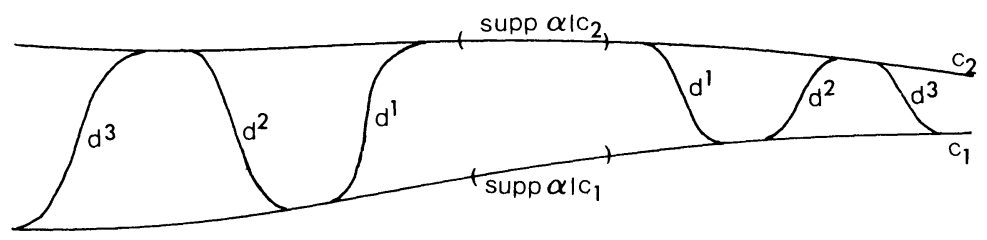

Observe that there exists a number $N$ such that for odd $s>N$

$$
\operatorname{supp}\left(\alpha \mid d^{s}\right)=\operatorname{supp}\left(\alpha \mid c_{2}\right) \text {. }
$$

It is so because if the contrary is true then we can build the submanifold

$$
b=\bigcup_{k=1}^{\infty} b^{k}
$$

where $b^{k}=\left\{\left[d^{k}-\left(d^{k} \cap c_{1}\right)\right]-\left(d^{k} \cap c_{2}\right)\right\} \cup\left(d^{k} \cap d^{k-1}\right) \cup\left(d^{k} \cap d^{k+1}\right)$ for $k>1$ and $b^{1}=\left[d^{1}-\left(d^{1} \cap c_{1}\right)\right] \cup\left(d^{1} \cap d^{2}\right)$. 
It can be easily shown that $b$ satisfies axiom 2 . Thus $b \in \mathscr{C}$ and $\alpha \mid b$ has non-compact support which contradicts our assertion. So taking any odd $s>N$ we have $c=d^{s}$.

Observe that if $c_{1}, c_{2}$ lie in the same state $\Omega$ than $d^{k}$ can be taken in $\Omega$ (axiom 4). Thus $c$ lies also in $\Omega$.

Proof of Theorem 2: If $c_{1}, c_{2} \subset \Omega$ then take $c \in \Omega$ as in Lemma 4. Because $\left\langle c_{2}, \alpha\right\rangle=\langle c, \alpha\rangle$ we have $\left\langle c_{1}, \alpha\right\rangle-\left\langle c_{2}, \alpha\right\rangle=\left\langle c_{1}, \alpha\right\rangle-\langle c, \alpha\rangle$. Using the axiom 6 we can take the field $X$ joining $c_{1}$ with $c$. The family $c_{t}=G_{t}^{X}(c)\left(c_{0}=c\right)$ "sweeps" the $n$-dimensional (possibly degenerated) compact volume $V \subset \Omega$ whose border is:

Thus

$$
\partial V=c_{1}-c .
$$

$$
\left\langle c_{1}, \alpha\right\rangle-\langle c, \alpha\rangle=\langle\partial V, \alpha\rangle=\langle V, d \alpha\rangle=0
$$

because $d \alpha \mid \Omega=0$ for any state $\Omega$.

Proof of Theorem 4: The observable $O$ generates the gauge field: $d \alpha=X\lrcorner \gamma, X \subset D$. Take any $c_{1}, c_{2} \in \mathscr{C}$ and $c \in \mathscr{C}$ as in Lemma 4. Using the same arguments as in precedent proof we find

$$
\left\langle c_{1}, \alpha\right\rangle-\left\langle c_{2}, \alpha\right\rangle=\left\langle c_{1}, \alpha\right\rangle-\langle c, \alpha\rangle=\langle\partial V, \alpha\rangle=\langle V, d \alpha\rangle
$$

for some $n$-dimensional (possibly degenerated) compact volume $V$ "swept" by the family $\left(c_{t}\right)$ of a.i.s. But every $n$-vector tangent to $V$ can be written as $u=u_{n-1} \wedge Y$, where $u_{n-1}$ is tangent to some a.i.s. $c_{t}$. Thus

$$
\left.\left.\langle u, d \alpha\rangle=\left\langle u_{n-1} \wedge Y, X\right\lrcorner \gamma\right\rangle=-\left\langle u_{n-1} \wedge X, Y\right\lrcorner \gamma\right\rangle=0
$$

because $u_{n-1} \wedge X$ is tangent to some state $\Omega$.

Proof of Theorem 3: It suffices to prove that the zero-observable generates only gauge-fields. Let $d \alpha=X\lrcorner \gamma$ and $\langle c, \alpha\rangle=0$ for any $c \in \mathscr{C}$. Suppose that there exists a point $p \in \mathscr{P}$ such that $X_{p} \notin D_{p}$. It means that there exists a state $\Omega$ passing by $p$, such that $X_{p}$ is not tangent to $\Omega$. Using the axiom 7 we see that the family of all $(n-1)$-vectors tangent to all a.i.s. passing by $p$ and lying in the same state $\Omega$ form a total set in $n-1$

$\bigwedge T_{p}(\Omega)$. Thus if we suppose $\left.\left.v\right\lrcorner\left(X_{p}\right\lrcorner \gamma_{p}\right)=0$ for all $(n-1)$-vectors $v$ tangent to all a.i.s. lying in $\Omega$ and passing by $p$, then the same is true for all $v \in \bigwedge^{n-1} T_{p}(\Omega)$. It would mean that $T_{p}(\Omega) \oplus\left\{\tau \cdot X_{p}\right\}$ is a singular space for $\gamma$ (i.e. belongs to $\left.\mathscr{A}_{p}^{\prime}\right)$. From the regularity condition for $(\mathscr{P}, \gamma)$ we derive: $X_{p} \in T_{p}(\Omega)$ which contradicts our assumption. Hence there exist an a.i.s. $c \in \mathscr{C}$ passing by $p$ and its tangent $(n-1)$-vector $v$ (at $p$ ) for which 
$\left.v\lrcorner\left(X_{p}\right\lrcorner \gamma_{p}\right) \neq 0$. Take such $Y_{p}$ that

$$
\begin{aligned}
\left.\left.0 \neq\left\langle Y_{p}, v\right\lrcorner\left(X_{p}\right\lrcorner \gamma_{p}\right)\right\rangle & \left.=\left\langle Y_{p} \wedge v, X_{p}\right\lrcorner \gamma_{p}\right\rangle \\
& \left.=(-1)^{n-1}\left\langle v,\left(Y_{p} \wedge X_{p}\right)\right\lrcorner \gamma_{p}\right\rangle .
\end{aligned}
$$

Take a vector field $Y$ which satisfies axiom 8 and $c_{t}=G_{t}^{Y}(c)$. Thus (cf. [6]):

$$
\left.\left.\left.\frac{d}{d t}\right|_{t=0}\left\langle c_{t}, \alpha\right\rangle=\int_{c} Y\right\lrcorner d \alpha=\langle c,(Y \wedge X)\lrcorner \gamma\right\rangle .
$$

For sufficiently small support of the field $Y$ it follows from (14) and the mean-value theorem that (15) is not equal zero. It means that $O_{\alpha}$ is not constant which contradicts our assumption.

\section{Appendix : Calculation of Canonical Fields in Scalar Field Theory}

For simplicity of computation take the Scalar Field Theory in 2-dimensional space-time (the identical result can be obtained for 4 dimensions):

$$
\left(\square+m^{2}\right) \varphi=\lambda \varphi^{n}
$$

$\square=\partial_{\mu} \partial^{\mu}=\left(\partial_{0}\right)^{2}-\left(\partial_{1}\right)^{2}, \mu=0,1$. In coordinates $\left(x^{\mu}, \varphi, \eta^{\mu}\right)$ the canonical forms $\omega$ and $\gamma$ are:

$$
\begin{gathered}
\omega=\left\{-\frac{1}{2}\left(\eta_{\mu} \eta^{\mu}+m^{2} \varphi^{2}\right)+\frac{1}{n+1} \lambda \varphi^{n+1}\right\} d x^{0} \wedge d x^{1} \\
+\eta^{0} d \varphi \wedge d x^{1}+\eta^{1} d x^{0} \wedge d \varphi \\
\gamma=d \omega=-\eta_{\mu} d \eta^{\mu} \wedge d x^{0} \wedge d x^{1}+Q(\varphi) d \varphi \wedge d x^{0} \wedge d x^{1} \\
+d \eta^{0} \wedge d \varphi \wedge d x^{1}+d \eta^{1} \wedge d x^{0} \wedge d \varphi,
\end{gathered}
$$

where $Q(\varphi)=\lambda \varphi^{n}-m^{2} \varphi$. Take any vector field

$$
X=A^{\mu} \frac{\partial}{\partial x^{\mu}}+B \frac{\partial}{\partial \varphi}+C^{\mu} \frac{\partial}{\partial \eta^{\mu}} .
$$

We shall solve the equation for canonical fields: $d(X\lrcorner \gamma)=0$. We obtain:

$$
\begin{aligned}
d(X\lrcorner \gamma)= & f_{1} d x^{0} \wedge d x^{1} \wedge d \varphi+f_{2} d x^{0} \wedge d x^{1} \wedge d \eta^{0}+f_{3} d x^{0} \wedge d x^{1} \wedge d \eta^{1} \\
& +f_{4} d x^{0} \wedge d \varphi \wedge d \eta^{0}+f_{5} d x^{0} \wedge d \varphi \wedge d \eta^{1}+f_{6} d x^{1} \wedge d \varphi \wedge d \eta^{0} \\
& +f_{7} d x^{1} \wedge d \varphi \wedge d \eta^{1}+f_{8} d x^{0} \wedge d \eta^{0} \wedge d \eta^{1}+f_{9} d x^{1} \wedge d \eta^{0} \wedge d \eta^{1} \\
& +f_{10} d \varphi \wedge d \eta^{0} \wedge d \eta^{1}=0
\end{aligned}
$$


where:

$$
\begin{aligned}
& f_{1}=Q \cdot\left(\partial_{\mu} A^{\mu}+\frac{\partial B}{\partial \varphi}\right)-\eta_{\mu} \frac{\partial C^{\mu}}{\partial \varphi}-\partial_{\mu} C^{\mu}+B \frac{\partial Q}{\partial \varphi} \\
& f_{2}=-\eta_{0}\left(\partial_{\mu} A^{\mu}\right)+Q \frac{\partial B}{\partial \eta^{0}}+\partial_{0} B-\eta_{\mu} \frac{\partial C^{\mu}}{\partial \eta^{0}}-C_{0} \\
& f_{3}=-\eta_{1}\left(\partial_{\mu} A^{\mu}\right)+Q \frac{\partial B}{\partial \eta^{1}}+\partial_{1} B-\eta_{\mu} \frac{\partial C^{\mu}}{\partial \eta^{1}}-C_{1} \\
& f_{4}=-\eta_{0} \frac{\partial A^{1}}{\partial \varphi}-Q \frac{\partial A^{1}}{\partial \eta^{0}}-\partial_{0} A^{1}+\frac{\partial C^{1}}{\partial \eta^{0}} \\
& f_{5}=-\eta_{1} \frac{\partial A^{1}}{\partial \varphi}-Q \frac{\partial A^{1}}{\partial \eta^{1}}+\partial_{0} A^{0}+\frac{\partial C^{1}}{\partial \eta^{1}}+\frac{\partial B}{\partial \varphi} \\
& f_{6}=\eta_{0} \frac{\partial A^{0}}{\partial \varphi}+Q \frac{\partial A^{0}}{\partial \eta^{0}}-\partial_{1} A^{1}-\frac{\partial C^{0}}{\partial \eta^{0}}-\frac{\partial B}{\partial \varphi} \\
& f_{7}=\eta_{1} \frac{\partial A^{0}}{\partial \varphi}+Q \frac{\partial A^{0}}{\partial \eta^{1}}-\partial_{1} A^{0}-\frac{\partial C^{0}}{\partial \eta^{1}} \\
& f_{8}=\eta_{0} \frac{\partial A^{1}}{\partial \eta^{1}}-\eta_{1} \frac{\partial A^{1}}{\partial \eta^{0}}+\frac{\partial B}{\partial \eta^{0}} \\
& f_{9}=\eta_{1} \frac{\partial A^{0}}{\partial \eta^{0}}-\eta_{0} \frac{\partial A^{0}}{\partial \eta^{1}}+\frac{\partial B}{\partial \eta^{1}} \\
& f_{10}=-\frac{\partial A^{1}}{\partial \eta^{1}}-\frac{\partial A^{0}}{\partial \eta^{0}} \cdot
\end{aligned}
$$

Let us introduce the following notation:

$$
P\left(x^{\mu}, \varphi, \eta^{\mu}\right):=\eta_{\mu} A^{\mu}=\eta_{0} A^{0}+\eta_{1} A^{1}=\eta^{0} A^{0}-\eta^{1} A^{1}
$$

(i)

$$
\begin{aligned}
& \frac{\partial P}{\partial \eta^{0}}=A^{0}+\eta^{0} \frac{\partial A^{0}}{\partial \eta^{0}}-\eta^{1} \frac{\partial A^{1}}{\partial \eta^{0}} \\
& \frac{\partial P}{\partial \eta^{1}}=-A^{1}-\eta^{1} \frac{\partial A^{1}}{\partial \eta^{1}}+\eta^{0} \frac{\partial A^{0}}{\partial \eta^{1}} .
\end{aligned}
$$

Using equations $f_{10}=0$ and $f_{8}=0$ we have

$$
\eta^{0} \frac{\partial A^{0}}{\partial \eta^{0}}=-\eta^{0} \frac{\partial A^{1}}{\partial \eta^{1}}=-\eta_{1} \frac{\partial A^{1}}{\partial \eta^{0}}+\frac{\partial B}{\partial \eta^{0}} .
$$


Putting it in (i) we have:

$$
A^{0}=\frac{\partial P}{\partial \eta^{0}}-\eta^{0} \frac{\partial A^{0}}{\partial \eta^{0}}+\eta^{1} \frac{\partial A^{1}}{\partial \eta^{0}}=\frac{\partial P}{\partial \eta^{0}}-\frac{\partial B}{\partial \eta^{0}}=-\frac{\partial}{\partial \eta^{0}}(B-P) .
$$

In similar way we obtain from (ii): $A^{1}=\frac{\partial}{\partial \eta^{1}}(B-P)$. Denoting $F:=B-P$ we have:

$$
A_{\mu}=-\frac{\partial}{\partial \eta^{\mu}} F .
$$

Now we try to express all variables by terms of $F$ :

$$
\begin{gathered}
P=\eta_{\mu} A^{\mu}=-\eta_{\mu} \frac{\partial}{\partial \eta^{\mu}} F, \\
B=F+P=F-\eta^{\mu} \frac{\partial}{\partial \eta^{\mu}} F .
\end{gathered}
$$

If we take $\frac{\partial C^{0}}{\partial \eta^{0}}$ from equality $f_{6}=0$ and $\frac{\partial C^{1}}{\partial \eta^{0}}$ from $f_{4}=0$ and put them into equality $f_{2}=0$ we obtain

$$
\begin{aligned}
C_{0}= & -\eta_{0}\left(\partial_{\mu} A^{\mu}\right)+Q \frac{\partial B}{\partial \eta^{0}}+\partial_{0} B-\eta_{0}\left(\eta_{0} \frac{\partial A^{0}}{\partial \varphi}+Q \frac{\partial A^{0}}{\partial \eta^{0}}-\partial_{1} A^{1}-\frac{\partial B}{\partial \varphi}\right) \\
& -\eta_{1}\left(\eta_{0} \frac{\partial A^{1}}{\partial \varphi}+Q \frac{\partial A^{1}}{\partial \eta^{0}}+\partial_{0} A^{1}\right)=\partial_{0} F+\eta_{0} \frac{\partial F}{\partial \varphi} \\
& +Q\left(\frac{\partial F}{\partial \eta^{0}}+A_{0}\right)=\partial_{0} F+\frac{\partial F}{\partial \varphi} \cdot \eta_{0} .
\end{aligned}
$$

In similar way we can compute $C_{1}$. Finally

$$
C_{\mu}=\partial_{\mu} F+\eta_{\mu} \frac{\partial F}{\partial \varphi} .
$$

If we substract equations $f_{5}=0$ and $f_{6}=0$ then using equality $f_{10}=0$ we obtain

$$
\begin{aligned}
0= & \eta_{\mu} \frac{\partial A^{\mu}}{\partial \varphi}-\partial_{\mu} A^{\mu}-\frac{\partial C^{\mu}}{\partial \eta^{\mu}}-2 \frac{\partial B}{\partial \varphi}=-\eta_{\mu} \frac{\partial^{2} F}{\partial \eta^{\mu} \partial \varphi}+\partial^{\mu} \frac{\partial}{\partial \eta^{\mu}} F \\
& -\frac{\partial}{\partial \eta^{\mu}} \partial^{\mu} F-2 \frac{\partial F}{\partial \varphi}-\eta_{\mu} \frac{\partial^{2} F}{\partial \eta^{\mu} \partial \varphi}-2 \frac{\partial B}{\partial \varphi}=2 \frac{\partial}{\partial \varphi}\left(\eta_{\mu} A^{\mu}\right)-2 \frac{\partial F}{\partial \varphi} \\
& -2 \frac{\partial B}{\partial \varphi}=-4 \frac{\partial F}{\partial \varphi} .
\end{aligned}
$$


Hence $\frac{\partial}{\partial \varphi} F=0$ and further

Moreover

$$
\frac{\partial A^{\mu}}{\partial \varphi}=\frac{\partial B}{\partial \varphi}=\frac{\partial C^{\mu}}{\partial \varphi}=0 .
$$

$$
C_{\mu}=\partial_{\mu} F \text {. }
$$

From equations $f_{4}=0, f_{5}=0, f_{6}=0$, and $f_{7}=0$ we thus obtain $\frac{\partial A^{\mu}}{\partial \eta^{\nu}}=0$ because $Q \cdot \frac{\partial A^{\mu}}{\partial \eta^{v}}$ are there unique terms containing the variable $\varphi$. It means that $\frac{\partial^{2}}{\partial \eta^{\mu} \partial \eta^{v}} F=0$, so $F$ is linear in variables $\eta^{\mu}$ :

$$
F\left(x^{\nu}, \varphi, \eta^{\mu}\right)=-\eta_{\mu} A^{\mu}\left(x^{\nu}\right)+D\left(x^{\nu}\right)=D\left(x^{\nu}\right)-P\left(x^{\nu}, \eta^{\mu}\right) .
$$

If we compare it with the definition of $F$ we see that $D=B$. Thus

$$
F=-\eta_{\mu} A^{\mu}+B
$$

where $A$ and $B$ depend only on $x^{v}$. Now equation $f_{1}=0$ reads

$$
Q \cdot \partial_{\mu} A^{\mu}-\square F+B \frac{\partial Q}{\partial \varphi}=0 .
$$

But $Q$ and $\frac{\partial Q}{\partial \varphi}$ are polynomials of the variable $\varphi$ of different degrees.

Hence

$$
\partial_{\mu} A^{\mu}=0
$$

$$
\square F-B \frac{\partial Q}{\partial \varphi}=0 .
$$

Putting (v) into equality $f_{5}=0$ or $f_{6}=0$ we obtain $\partial_{0} A^{0}-\partial_{1} A^{1}=0$. Combining it with (vi) we have $\partial_{0} A^{0}=0, \partial_{1} A^{1}=0$. Thus

$$
A^{0}=f\left(x^{1}\right) \quad A^{1}=g\left(x^{0}\right) .
$$

Putting (v) into equality $f_{4}=0$ or $f_{7}=0$ we get $\partial_{0} A^{1}-\partial_{1} A^{0}=0$. It means that $f$ and $g$ must be linear functions of their arguments and:

$$
\begin{gathered}
A^{0}\left(x^{1}\right)=f\left(x^{1}\right)=a x^{1}+b, \quad A^{1}\left(x^{0}\right)=g\left(x^{0}\right)=a x^{0}+c, \\
F=-\eta_{0}\left(a x^{1}+b\right)-\eta_{1}\left(a x^{0}+c\right)+B, \\
C_{0}=\partial_{0} F=-a \eta_{1}+\partial_{0} B, \quad C_{1}=\partial_{1} F=-a \eta_{0}+\partial_{1} B, \\
C^{0}=a \eta^{1}+\partial^{0} B, \quad C^{1}=a \eta^{0}+\partial^{1} B .
\end{gathered}
$$


Putting $B=0$ we obtain 3-dimensional algebra of Poincaré group ( $b$ and $c$ give translations and $a$-Lorentz rotation).

Now let us turn to Eq. (vii). Because of equality $\square A=0$ it reads:

$$
B+B\left(m^{2}-\lambda n \varphi^{n-1}\right)=0 .
$$

For the linear case $(\lambda=0)$ we obtain $\left(\square+m^{2}\right) B=0$ and for $a=b=c=0$ we have

$$
X=B \frac{\partial}{\partial \varphi}+\partial^{\mu} B \frac{\partial}{\partial \eta^{\mu}} .
$$

For non-linear case $(\lambda \neq 0) B=0$ and the only canonical fields are Poincaré fields.

\section{References}

1. Bergman, P.G.: Status of canonical quantization, preprint.

2. Białynicki-Birula,I., Białynicka-Birula,Z.: Elektrodynamika Kwantowa, Warszawa: Państwowe Wydawnictwo Naukowe 1969.

3. Białynicki-Birula, I.: Gauge invariant canonical formalism, Reports on Math. Phys. 1, $83-86(1970)$.

4. Białynicki-Birula, I.: Solutions of the Equations of Motion in Classical and Quantum Theories. Ann. Phys. 67, (1) 252-273 (1971).

5. Bishop, R.L., Critenden, R.J.: Geometry of Manifolds. New York: Academic Press 1964.

6. Dedecker,P.: Calcul des variations, formes différentielles et champs géodésiques, Colloque International de Géometrie Différentielle. Straßbourg 1953.

7. Eells, J., Jr.: On the geometry of function spaces. Symp. Inter. de Topologia Alg., Mexico 303-308, 1956-1958.

8. Gawedzki,K.: On the geometrization of the canonical formalism in the classical field theory, Reports on Math. Phys. (in print).

9. Kijowski,J.: Existence of differentiable structure in the set of submanifolds. Studia Math. 33, 93-108 (1969).

10. Kijowski, J.: On representation of functionals of local type by differential forms, Colloquium Mathematicum, 26, 293-312 (1972).

11. Marsden, J.E.: Generalized Hamiltonian Mechanics. Arch. Rat. Mech. Anal. 28, $323-361$ (1968).

12. Marsden, J.E.: Hamiltonian One Parameter Groups. Arch. Rat. Mech. Anal. 28, $362-394$ (1968).

13. Maurin, K.: Analiza, Warszawa: Państwowe Wydawnictwo Naukowe 1971.

14. Segal, J.E.: Mathematical Problems of Relativistic Physics, Proceedings of the Summer Seminar, Boulder, Colorado, 1960.

15. Tulczyjew, W.M.: Seminar on Phase SpaceTheory. Warsaw 1968 (unpublished).

16. Tulczyjew, W.M., Sniatycki, J.: Canonical dynamics of relativistic charged particles. Ann. Inst. H. Poincaré A, 15, 177-187 (1971).

J. Kijowski

Institute of Mathematical Methods of

Physics

ul. Hoza 69

Warsaw, Poland 